\title{
The effect of the time interval between the last breastfeeding and Tc-99m MIBI injection on the intensity of Tc-99m MIBI uptake in breast tissue
}

\author{
Bekir Tasdemir $^{1^{*}}$, Zeki Dostbil ${ }^{1}$, Ilhan Sezgin ${ }^{1}$
}

\section{Abstract}

In this case report, we present a 36-year-old lactating woman, 10 months after delivery, who was referred to our clinic for a myocardial perfusion scintigraphy (MPS) due to chest pain. A scintigraphic procedure was performed according to the two-day protocol. On the first day, dipyridamole pharmacological stress Tc-99m MIBI MPS was performed, and on the second day, the same test was repeated while in a resting state without the administration of any pharmacological agent. The stress scan, compared to the rest scan, presented a higher uptake of Tc-99m MIBI in the breast tissue. The patient query revealed that the last breastfeeding was 12 hours before the stress scan and she did not breastfeed or express breast milk prior to the rest scan. Our findings suggest that ceasing the emptying of the mammary glands prior to the Tc-99m MIBI injection might be appropriate to decrease the radiation exposure of the mammary glands and the breast-fed baby.

Key words: molecular imaging, myocardial perfusion imaging, SPECT, technetium Tc 99m sestamibi

\section{Introduction}

Technetium-99m (Tc-99m) 2-methoxy-2methyl-isopropyl-1-isonitrile (MIBI) is one of the most common radiopharmaceuticals used in myocardial perfusion scintigraphy. Tc-99m MIBI concentrates in the myocardium in proportion to myocardial blood flow and shows slow washout and redistribution (1). Tc-99m MIBI is a lipophilic cationic agent that mainly penetrates into the mitochondrial compartment as a response to the high negative transmembrane potential in the myocytes (2). However, Tc-99m MIBI is not unique to the myocardial cells and may also concentrate in other tissues and organs (3). Breast tissue in lactating women is one of these tissues where diffuse Tc $99 \mathrm{~m}$ MIBI uptake tends to concentrate (4, 5). In this case report, we present a lactating woman whose clinical profile indicated that the time interval between the last breastfeeding and the Tc-99m MIBI injection might affect the intensity of Tc-99m MIBI uptake in her breast tissue

\section{Case}

A 36-year-old lactating woman, 10 months after delivery, was admitted to our cardiology clinic due to chest pain. In line with her clinical history, the chest pain was considered to be of cardiac origin. Although the electrocardiogram was normal, the patient was required to undergo an exercise stress test since she presented with typical cardiac symptoms.
However, the patient refused to take the treadmill exercise stress test due to her orthopaedic problems and thus was referred to our clinic for myocardial perfusion imaging with pharmacological stress. Scintigraphic procedures were performed according to the two-day protocol.

On the first day, pharmacological stress imaging with dipyridamole was performed, and on the second day, the test was repeated in a resting state without the administration of any pharmacological agent. The patient fasted for a minimum of four hours prior to both the stress and rest scans. For each scan, $15 \mathrm{mCi}(555 \mathrm{MBq})$ of Tc-99m MIBI was injected intravenously and the scans began 30 minutes following the injections.

The scans were performed using a Philips Brightview dual-headed gamma camera (Philips Medical Systems, Inc., Cleveland, $\mathrm{OH}$, USA), and low-energy general purpose collimators were used for the imaging.

The patient was placed on the table in the supine position and perfusion single-photon emission computed tomography (SPECT) acquisition was performed using a $180^{\circ}$ circular orbit, starting at $45^{\circ}$ right anterior oblique and ending at $45^{\circ}$ left posterior oblique. The patient's myocardial perfusion scintigraphy (MPS) was evaluated as normal. 


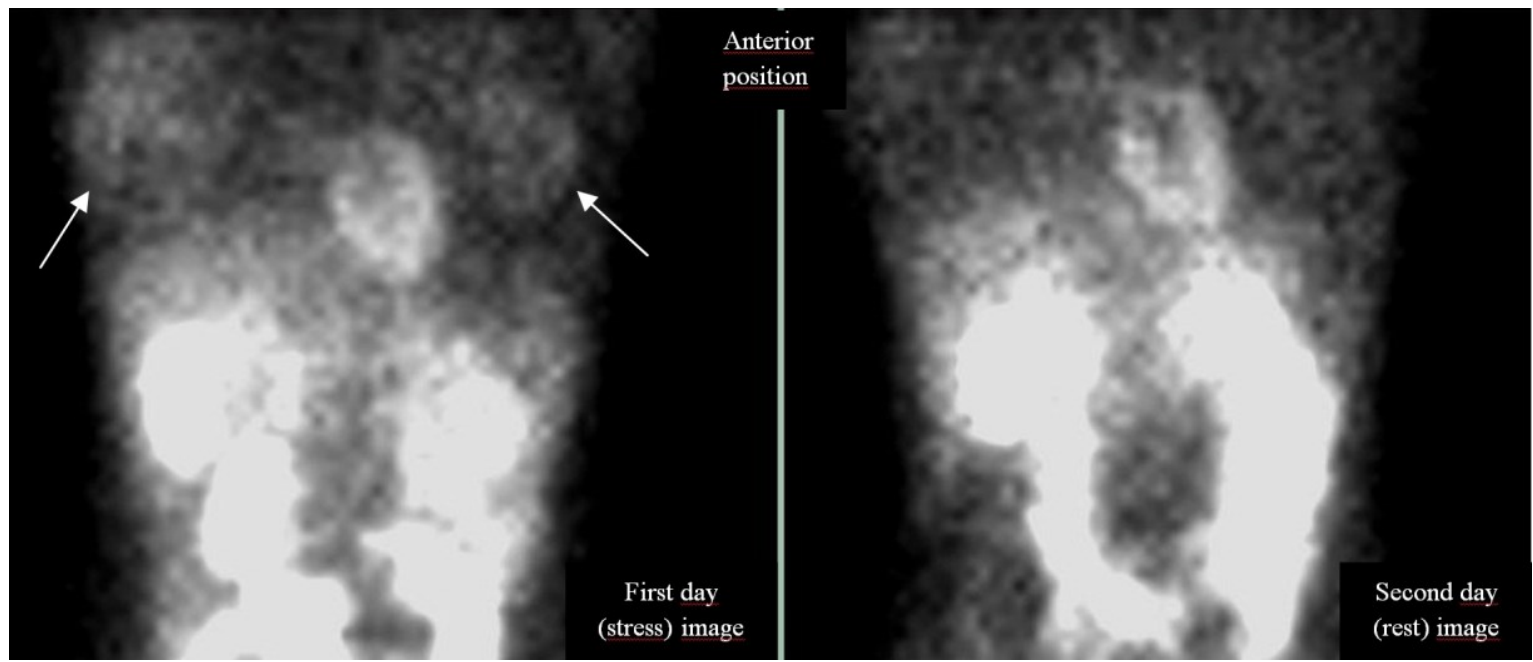

Figure 1: Tc-99m MIBI uptake in both breast tissues (arrows) is higher on the first-day (stress) image than on the second-day (rest) image.

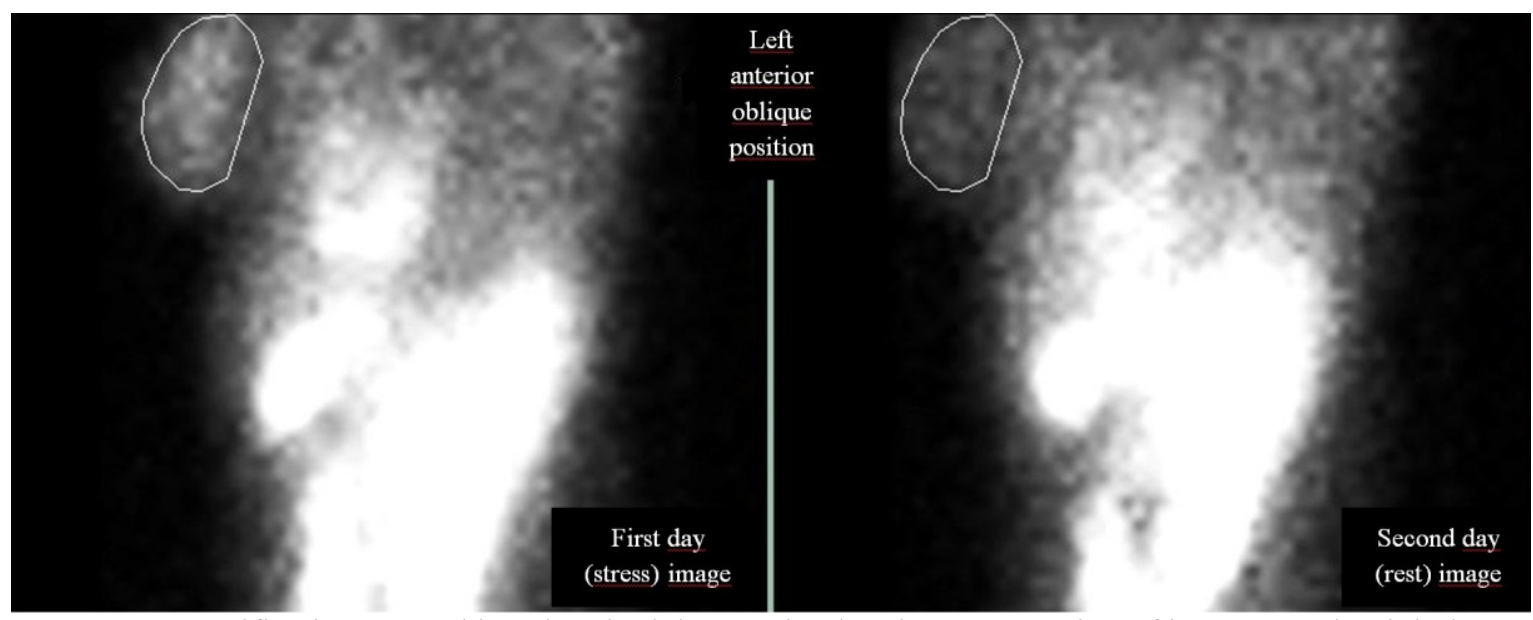

Figure 2: Quantification was achieved on both images by drawing same region of interest on the right breast tissue, and $88 \%$ higher Tc-99m MIBI uptake was measured on the first-day (stress) image compared with the second-day (rest) image.

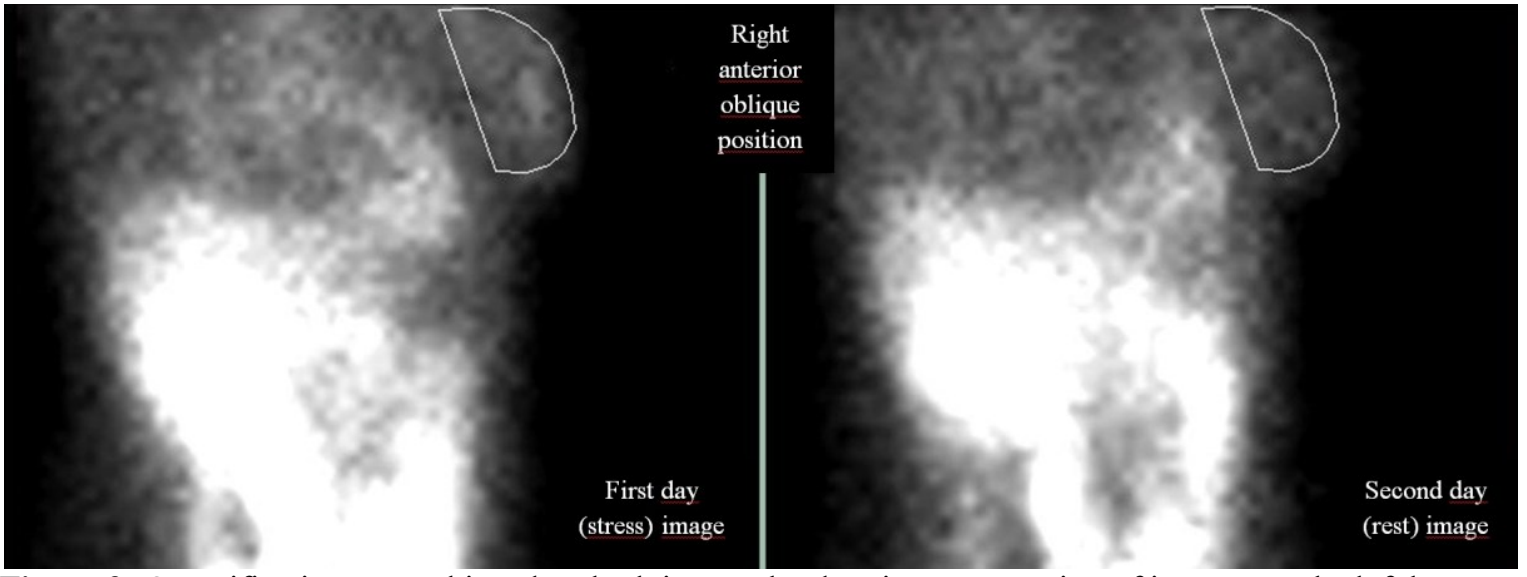

Figure 3: Quantification was achieved on both images by drawing same region of interest on the left breast tissue, and 52\% higher Tc-99m MIBI uptake was measured on the first-day (stress) image compared with the second-day (rest) image. 
However, it drew our attention that the firstday scan, compared to the second-day scan, presented a higher uptake of Tc-99m MIBI in the breast tissue, particularly in the right breast, when we were evaluating the raw data images (Figure 1).

Quantification was achieved by drawing a region of interest (ROI), including the glandular regions, on both breasts (Figures 2 and 3). During the quantification, in order to distinguish the breast tissues from other surrounding tissues, the right breast was evaluated on the left anterior oblique image and the left breast was evaluated on the right anterior oblique image (Figures 2 and 3).

The results of the quantification revealed that the Tc-99m MIBI uptake in the breast on the stress images was $88 \%$ higher in the right breast and $52 \%$ higher in the left breast than that of the images taken at rest. To investigate this situation, the patient was queried about the last breastfeeding time, and she revealed that the last breastfeeding was 12 hours before the stress scan and thus, 36 hours prior to the rest scan. We also determined that the patient had not taken any drugs, undertaken any invasive/noninvasive procedures related to the breast tissue, or undergone any extraordinary events in her daily life before the scanning processes. To prevent the baby from incurring any radiation exposure, the patient had been advised not to breastfeed her baby within the 24 hours following the stress scan and also to express her breast milk and discard it.

The patient stated that she did not breastfeed her baby during that period, as advised, however, she did not express and discard her breast milk. This means that the patient did not empty her mammary glands within the 36 hours prior to the rest scan. This situation was considered as a possible reason for the decrease in the Tc $99 \mathrm{~m}$ MIBI uptake on the rest images and also for the difference between the intensity of Tc-99m MIBI uptake of the right and left breasts on stress images. On the other hand, the patient was also asked as to which breast she had last used for breastfeeding, unfortunately, no data was obtained since she could not remember which breast was used. We also calculated the liver to lung uptake ratios of Tc-99m MIBI in the stress and rest images to rule out the potential for dipyridamole to change the biodistribution of Tc-99m MIBI. However, we did not find any significant difference between the uptake ratios of the stress (2.324) and rest (2.321) images

\section{Discussion}

In this case, we observed different intensities of Tc-99m MIBI uptake in breast tissue on two different days. We considered that this difference was primarily concerned with the time interval between the last breastfeeding and the Tc-99m MIBI injection as we ruled out the majority of the other possibilities in line with the patient's responses to our queries. The possible mechanism of the increased Tc-99m MIBI uptake in the breast tissue may be that breast alveoli may be filled with new milk early after the breastfeeding (6) and, this situation may be cause of the increased Tc-99m MIBI uptake in the breast tissue in our case. As another possibility, we also focused on the dipyridamole, which was administered for the pharmacological stress testing. However, in our literature review, we found no information that dipyridamole may increase Tc-99m MIBI uptake in breast tissue. We also did not find any significant difference between the liver to lung uptake ratios of the stress and rest images in our calculations.

It has been reported that the radiation exposure of a patient's baby should be avoided by ceasing any breastfeeding for a certain period of time following scintigraphic procedures, and that the breast milk should be expressed and discarded during this period (7).

However, Rubow et al. studied the retention and secretion of 11 different radiopharmaceuticals in breast tissues and reported that, among all of them, Tc-99m MIBI is the lowest excreted radiopharmaceutical in breast milk (5). As a result of our findings, in order to decrease the radiation exposure of both the mammary glands and the breastfed baby, the mother should cease emptying the mammary glands for a certain period of time prior to a Tc-99m MIBI injection. We feel this may be more appropriate than expressing and discarding the breast milk following the scanning procedure with Tc-99m MIBI. Moreover, this hypothesis might also be valid for other pharmaceuticals such as F-18 FDG (2fluoro-2-deoxy-D-glucose) and Tc-99m tetrofosmin, both of which have shown high uptakes in breast tissues of lactating women $(8,9)$

In conclusion, ceasing the emptying of mammary glands prior to the Tc-99m MIBI injection might be appropriate in order to decrease the radiation exposure of maternal mammary glands and the breastfed baby. Nevertheless, additional more detailed prospective studies are needed to confirm this hypothesis and to determine the appropriate time for ceasing the breastfeeding prior to a Tc-99m MIBI injection

\section{Acknowledgments: None}

Conflict of Interest: The authors declare no potential conflicts of interest with respect to the research, authorship, and/or publication of this article.

\section{References}

1. Okada RD, Glover D, Gaffney T, Williams S. Myocardial kinetics of technetium-99m-hexakis-2-methoxy-2methylpropyl-isonitrile. Circulation. 1988;77(2):491-8. 
2. Piwnicaworms D, Kronauge JF, Chiu ML. Uptake and retention of hexakis (2-methoxyisobutyl isonitrile) technetium(i) in cultured chick myocardial-cells mitochondrial and plasma-membrane potential dependence. Circulation. 1990;82(5):1826-38.

3. Mattsson S, Johansson L, Leide-Svegborn S, Liniecki J, Nosske D, Riklund K, et al., editors. Current activities in the ICRP concerning estimation of radiation doses to patients from radiopharmaceuticals for diagnostic use. International Conference on Image Optimisation in Nuclear Medicine (OptiNM); 20112011 Mar 23-26; Cyprus2011.

4. Ramakrishna G, Miller TD. Significant breast uptake of Tc-99m sestamibi in an actively lactating woman during SPECT myocardial perfusion imaging. Journal of Nuclear Cardiology. 2004;11(2):222-3.

5. Rubow S, Klopper J, Wasserman H, Baard B, Vanniekerk $M$. The excretion of radiopharmaceuticals in human breastmilk - additional data and dosimetry. European Journal of Nuclear Medicine. 1994;21(2):144-53.
6. Spinka M, Illmann G, Algers B, Stetkova Z. The role of nursing frequency in milk production in domestic pigs. Journal of Animal Science. 1997;75(5):1223-8.

7. Mountford PJ, Coakley AJ. A review of the secretion of radioactivity in human-breast milk - data, quantitativeanalysis and recommendations. Nuclear Medicine Communications. 1989;10(1):15-27.

8. Hicks RJ, Binns D, Stabin MG. Pattern of uptake and excretion of F-18-FDG in the lactating breast. Journal of Nuclear Medicine. 2001;42(8):1238-42.

9. Yamamoto W, Shuke N, Usui K, Aburano T, Takehara N, Kikuchi K. Intense uptake of technetium-99m tetrofosmin by lactating breasts. Clinical Nuclear Medicine. 2001;26(1):76-7.

Copyright (C) 2014 The Author(s); This is an open-access article distributed under the terms of the Creative Commons Attribution License (http://creativecommons.org/licenses/by/4.0), which permits unrestricted use, distribution, and reproduction in any medium, provided the original work is properly cited. All Rights reserved by international journal of Medical Science and Discovery. 